\title{
Internal insulation retrofit with ventilated wall and circulation of dry air - focus on airflow distribution and mitigation of noise
}

\author{
Christian A. Hviid ${ }^{1,2,}{ }^{*}$, Dessy Wina Harjani ${ }^{1}$, and Fabricio Lucchesi ${ }^{1}$ \\ ${ }^{1}$ Department of Civil Engineering, Technical University of Denmark, Brovej building 118, DK-2800 Kgs. Lyngby, Denmark \\ ${ }^{2}$ Saint-Gobain Nordic, Robert Jacobsens Vej 62A, DK-2300 Copenhagen S, Denmark
}

\begin{abstract}
External façade retrofit changes the look, feel and aesthetics of the building and internal façade retrofit changes the heat and moisture conditions of the existing façade. The ventilated internal insulation wall is an existing solution for internal retrofit applications that promises to be an efficient and moisture resilient retrofit solution. It has a ventilated cavity and the moisture content in the cavity is actively controlled by a small dehumidifier unit mounted in the wall. The dehumidifier circulates air inside the air cavity and any excessive moisture is ejected into the room air. The focus of the paper is on mitigating noise from the dehumidifer by analyzing the required airflow rate, the air distribution inside the air cavity as well as optimizing the dehumidifier. CFD simulations were carried out to analyze airflow velocities and distribution and the final results show a possible $30 \%$ reduction in the airflow rate without compromising the drying performance. In addition, experimental studies in noise mitigation were performed. Different dehumidifier designs with different fan configurations were tested. A significant noise reduction was obtained from $39 \mathrm{~dB}(\mathrm{~A})$ to a noise level of approx. $26 \mathrm{~dB}(\mathrm{~A})$.
\end{abstract}

\section{Introduction}

Internal insulation is particularly problematic in heritage buildings because they are often made from combinations of wood and stone materials. When applying internal insulation, the old façade is exposed to moisture from both inside and outside which may cause moisture build-up, and subsequent decay, fungi growth and rot of any organic materials [1].

The ventilated internal insulation wall is an existing solution for internal retrofit applications that promises to be an efficient and moisture resilient retrofit solution. The basic idea is to apply extra wall layers to the original (existing) wall to improve the insulation properties. In order to deal with moisture related problems, a dehumidifier unit is added to the system. The dehumidifier has the important role of ensuring adequate circulation of air in the cavity between the original wall and the extra wall layers in order to avoid the build-up of moist. The dehumidifier is of a desiccant type which is suitable for use in the winter time for areas with low temperatures and high relative humidity $(\mathrm{RH})$ like in Denmark. This solution is acceptable for commercial buildings, but for residential use, the solution generates too much noise. The main source of sound is the dehumidifier unit, in particular the fans. The air distribution ductwork and the layout also influences the noise level due to its impact on the air flow.

The major issue with ventilated internal insulations walls is the proximity of dehumidifier unit to the living quarters. Especially in dwellings and bedrooms, the noise from the unit must be addressed.

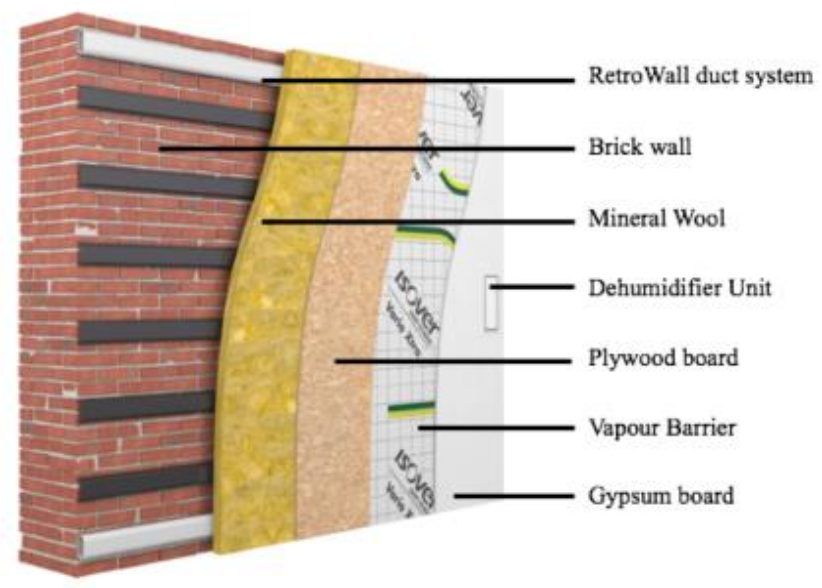

Fig. 1. Ventilated insulation wall system

Consequently, this paper shows the results of a focused effort on mitigating the noise from an existing solution. The objectives were to:

- Optimize airflow distribution inside the cavity wall in order to identify how much the flow rate could be reduced

- Reduce sound pressure level from $39 \mathrm{~dB}(\mathrm{~A})$ to 25 $\mathrm{dB}(\mathrm{A})$ by prioritized actions: a) reduce flow rate, $\mathrm{b}$ ) optimize fan configuration and flow path, c) apply muffling material, and d) enlarge the dehumidifier unit.

\footnotetext{
* Corresponding author: $\underline{\text { cah@byg.dtu.dk }}$
} 
We selected $25 \mathrm{~dB}(\mathrm{~A})$ as the target from the very low Swedish class A in dwellings [2].

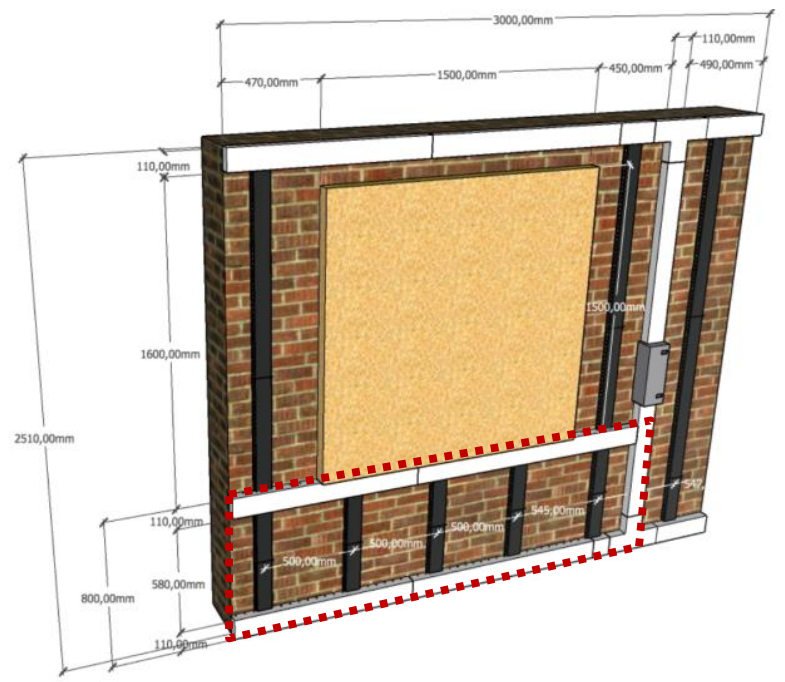

Fig. 2. Vertical and horizontal air distribution ducts (white) with dehumidifier unit (grey box on vertical duct). Dashed red square marks the targeted area for air flow optimization

\section{Internal ventilated insulation concept}

The ventilated insulation wall has a cavity of $25 \mathrm{~mm}$ where dry air flows from the top to the bottom. The air flows though the ducts in the wall and is dried in the dehumidifier. The ducts have the dimensions $54 \times 110$ $\mathrm{mm}$. The cavity is followed by $50 \mathrm{~mm}$ of mineral wool insulation, a $16 \mathrm{~mm}$ plywood board and finishes with a $13 \mathrm{~mm}$ gypsum board, as shown in Fig 1. The structure of the wall is supported by horizontal steel brackets (23x100 mm) which allow the air to pass. A desiccant dehumidifier provides moisture control keeping the relative humidity about $50 \%$, which prevents moisture related problems such as mould growth and wood decay. The dehumidifier unit is split into two sections of airflow: process air and regeneration air. The process air flows inside the air cavity and the regeneration air removes the absorbed moisture from the desiccant wheel and ejects it back into the room.

\subsection{Process air flow rate}

The heat, mass and air balance influences the necessary airflow rate to maintain the desired levels of relative humidity inside the cavity. To set up the moisture balance and calculate the equilibrium state of the system, climate data, internal and external temperatures, and relative humidity needs to be established. A brick wall exposed to wind driven rain will absorb moisture and the capillary suction will make water accumulate into the brick wall; hence the relative humidity within the wall will increase and result in a waterfront. When insulating a wall with vapour barrier on the inside, the moisture can no longer be diffused to the interior. Because of the colder wall surface, the vapour pressure difference between interior and the exterior will become very little, which will result in accumulation of moisture and thus longer periods of moist walls.

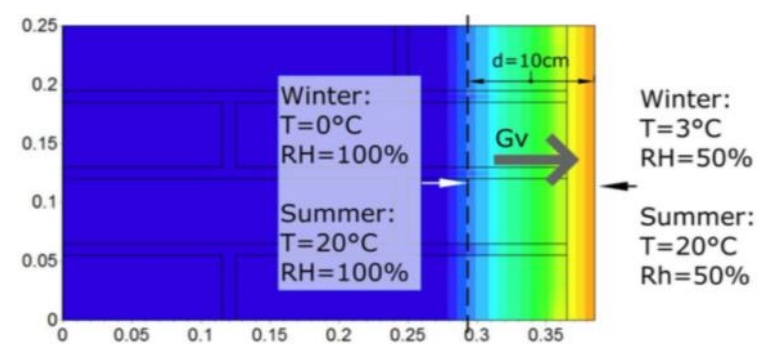

Fig. 3. Temperature, relative humidity and waterfront (dashed line) for winter and summer conditions for a North-oriented brick wall with internal insulation applied

Fig. 3 shows the temperature field based on climate data and simulations made in Delphin by Fosso [3]. From the summer and winter scenarios in Fig. 3, the diffusion, hence necessary drying capacity is calculated from Fick's law and presented in Table 1.

Table 1. Vapour diffusion and necessary drying capacity

\begin{tabular}{|c|c|}
\hline Season & $\begin{array}{c}\text { Vapour } \\
\text { diffusion }\end{array}$ \\
\hline Summer & $0.53 \mathrm{~g} /\left(\mathrm{m}^{2} . \mathrm{h}\right)$ \\
\hline Winter & $0.12 \mathrm{~g} /\left(\mathrm{m}^{2} . \mathrm{h}\right)$ \\
\hline
\end{tabular}

To ensure that moisture never exceeds $70 \%$ of relative humidity a minimum airflow rate $Q_{\min }$ is given by Equation 1, where $G_{\mathrm{vp}}$ is the diffusion per $\mathrm{m}^{2}$ of wall. Moisture content $(M C)$ for relative humidity of $70 \%$ and $50 \%$ are taken from the Mollier diagram. With a good margin the minimum airflow rate for summer and winter is then $0.2 \mathrm{~m}^{3} /\left(\mathrm{m}^{2} \cdot \mathrm{h}\right)$.

$$
Q_{\min }=G_{\mathrm{vp}} /\left[\rho_{\mathrm{air}}\left(M C_{70 \%}-M C_{50 \%}\right)\right]
$$

Previous, undocumented studies found a necessary airflow rate of $1.2 \mathrm{~m}^{3} /(\mathrm{m} 2 . \mathrm{h})$. Consequently, to be conservative, we allowed the design airflow rate to be reduced by $30 \%$ to $0.84 \mathrm{~m}^{3} /(\mathrm{m} 2 . \mathrm{h})$. For the size of the experimental mock-up, this corresponds to $11 \mathrm{~m}^{3} / \mathrm{h}$.

\section{Method}

To meet the objectives, the following methods were applied:

- CFD simulations used to target critical airflow distribution

- Testing of different dehumidifier designs and fan configurations, establishing pressure characteristics and measurements of noise

- Experimental verification of noise when mounted in mockup wall

The CFD simulations (computational fluid dynamics) were used to analyze airflow velocities and optimal distribution without compromising the drying 
performance. A 3D model using Ansys Fluent with the k-omega-sst turbulence model was used.

Several different configurations of axial and centrifugal fans, muffling materials and encapsulating boxes were tested in experimental prototypes. The internal parts of the dehumidier were 3D-printed and Fig. 4 and Fig. 5 depict prototypes in acrylic and metal boxes. Airflow rate and total pressure capability were measured at different fan speeds before the prototypes were mounted in the wall for noise measurements. The airflows and pressures were measured using a pitot tube and a micromanometer from Furness Controls FC510. A damper mounted downstream of the pitot tube was used to emulated different pressure resistances of the ductwork and air cavity inside the wall. The fan speed was controlled by the voltage signal.

Measurements of noise from dehumidifier unit were performed at a distance of $1 \mathrm{~m}$ horizontally from the dehumidifier when it was mounted in the mock up wall. Noise was measured using a Brüel \& Kjær Sound Level Analyzer type 2250 and 2240.

Only the results related to the final prototype is reported here. For more information, the reader is kindly referred to Harjani [5].

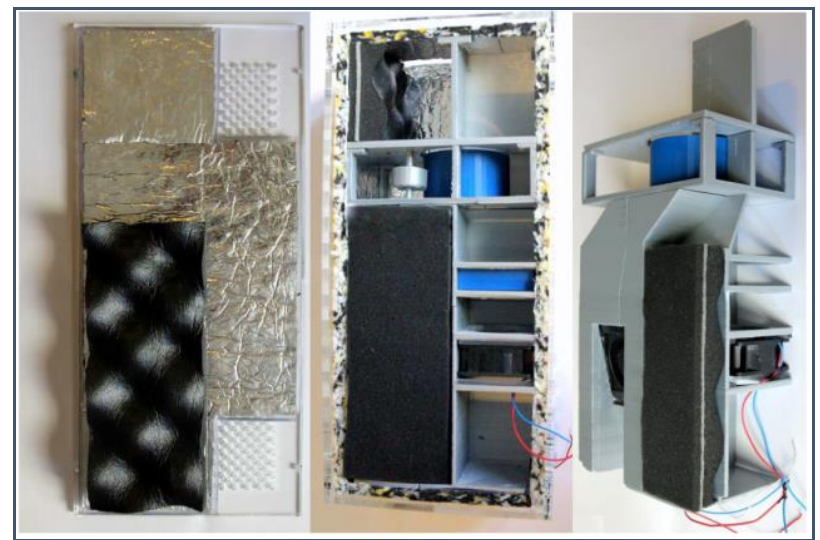

Fig. 4. Photos of the dehumidifier prototype in acrylic box with muffling material applied [5]

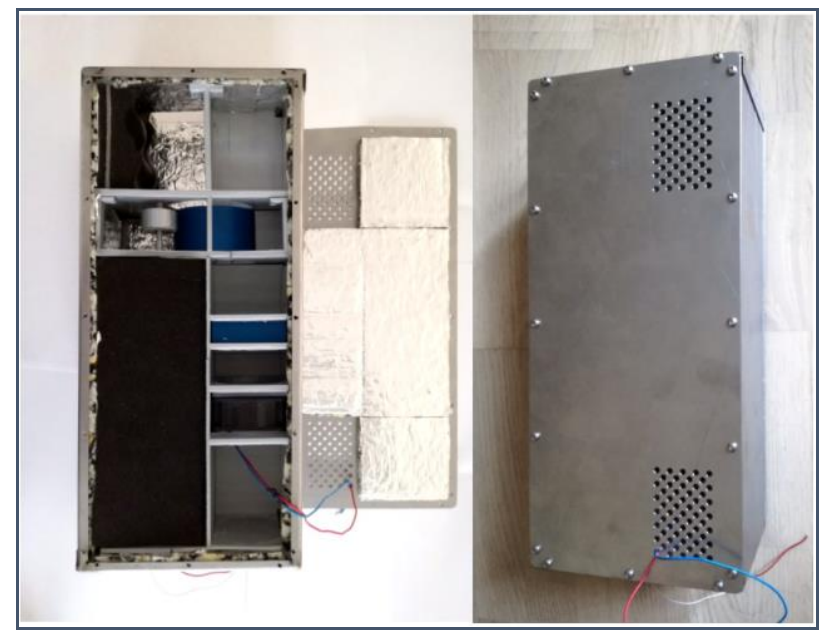

Fig. 5. Photos of the dehumidifier prototype in metal box [5]

\section{Results and discussion}

\subsection{Airflow optimization}

Fig. 6 illustrates the airflow pattern inside the cavity of the wall. It is clear that the part below the window is critical in terms of drying performance.

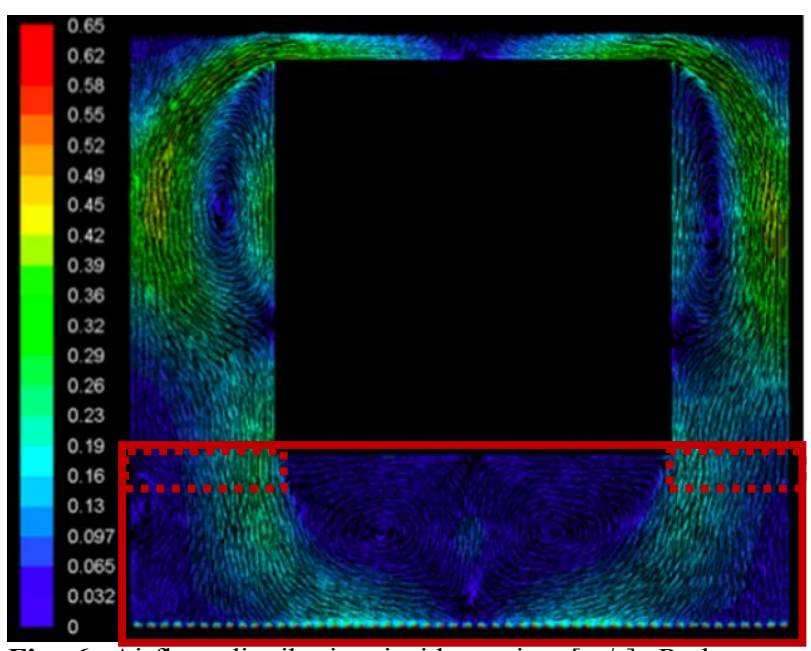

Fig. 6. Airflow distribution inside cavity, $[\mathrm{m} / \mathrm{s}]$. Red square marks the targeted area for improving the air distribution [4]

Consequently, we applied two pieces of duct (Fig. 7) to redirect part of the airflow to the critical section below the window. It shows clear that the critical parts are now ventilated. Also, the pressure drop of the duct system including these modifications was calculated to be approx. $3 \mathrm{~Pa}$ at $11 \mathrm{~m} 3 / \mathrm{h} \mathrm{[4].}$

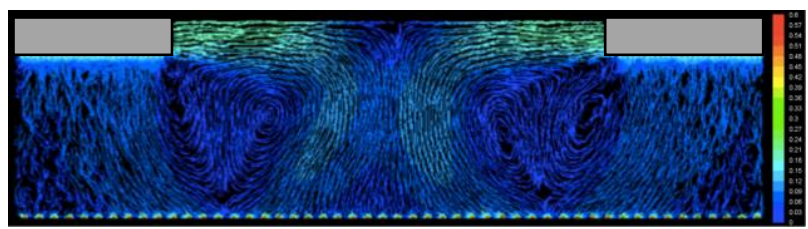

Fig. 7. Improved airflow distribution below window (red dashed squares in Fig. 4) [4]

\subsection{Dehumidifier units}

For the final prototype, the pressure characteristic is depicted in Fig. 8, which shows the relation between airflow rate and total pressure for three fan speeds (denoted by $8-10-12 \mathrm{~V}$ ). The final fan configuration was to use a centrifugal fan capable of higher pressure for the process air and a weaker axial fan with less pressure capability for the regenerative air. In order to accommodate the slightly bigger centrifugal fan we had to increase the width of the unit by $15 \mathrm{~mm}$. The dashed line in Fig. 8 indicates the pressure and fan speed necessary to move the design airflow rate of $11 \mathrm{~m}^{3} / \mathrm{h}$. 


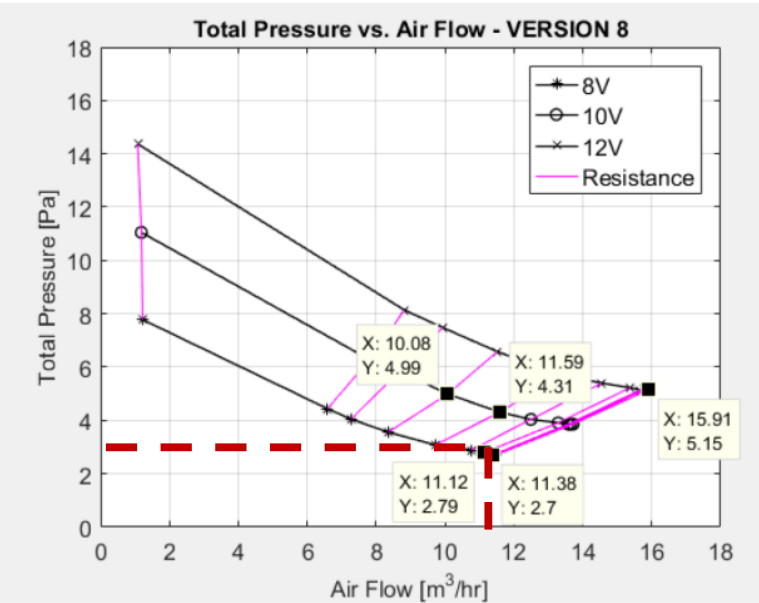

Fig. 8. Pressure characteristic for dehumidifier unit on process air side. 8-10-12V denotes different fan speeds [5].

\subsection{Sound pressure level}

The dehumidifier unit was mounted in the wall before the sound pressure level was measured. Fig. 9 depicts the sound pressure level of the new prototypes in comparison with the original dehumidifier. The noise reduction is partly due to reduced airflow rate, selection of quitter fans more suitable for the task, smoothening of the air path inside the dehumidifier unit and the application of muffling material in the cavities of the dehumidifier unit.

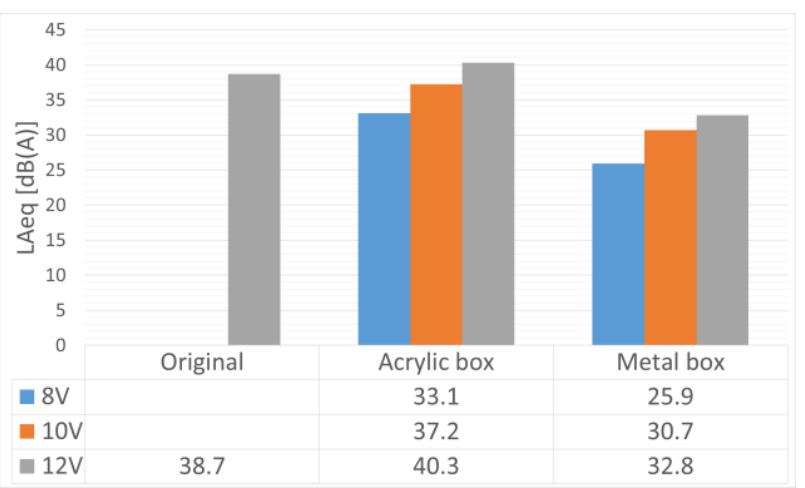

Fig. 9. Sound pressure level at different fan speeds (8-12V)

\section{Conclusion}

The objective was to mitigate the noise issue from a commercially available ventilated insulation wall for retrofit of heritage buildings. The objective was to reduce the sound pressure level below $25 \mathrm{~dB}(\mathrm{~A})$ and with $25.9 \mathrm{~dB}(\mathrm{~A})$ we almost achieved this goal.

We achieved this goal by questioning the required airflow rate, targeting the critical airflow sections, optimizing the fan configuration and smoothening the flow paths inside the dehumidifier unit. Eventually, the ultimate goal is to achieve $20 \mathrm{~dB}(\mathrm{~A})$ which is considered completely inaudible in bedrooms. This could be achieved by reducing the airflow further to the calculated design airflow, but it will require more experimental testing of the long-term drying performance under real operating conditions.

\section{References}

1. T. Odgaard, S.P. Bjarløv, C. Rode, M. Vesterlokke, 6th International Building Physics Conference, IBPC 78 830-835 (2015)

2. Svensk Standard, Byggakustik - Ljudklassning av utrymmen i bygnader - Bostäder, SS 25267 (2015)

3. K. Fosso, M.Sc. thesis, Dept. of Civ. Eng., Tech. Univ. of Denmark (2016)

4. F.G. Lucchesi, M.Sc. thesis, Dept. of Civ. Eng., Tech. Univ. of Denmark (2018)

5. D.W. Harjani, M.Sc. thesis, Dept. of Civ. Eng., Tech. Univ. of Denmark (2018) 